\title{
On the Contact Dimensions of Graphs
}

\author{
P. Frankl ${ }^{1}$ and H. Maehara ${ }^{2}$
}

'CNRS, Paris, France

${ }^{2}$ Ryukyu University, Okinawa, Japan

\begin{abstract}
Every simple graph $G=(V, E)$ can be represented by a family of equal nonoverlapping spheres $\left\{S_{v}: v \in V\right\}$ in a Euclidean space $R^{n}$ in such a way that $u v \in E$ if and only if $S_{u}$ and $S_{v}$ touch each other. The smallest dimension $n$ needed to represent $G$ in such a way is called the contact dimension of $G$ and it is denoted by $\operatorname{cd}(G)$. We prove that $(1) \operatorname{cd}(T)<(7.3) \log |T|$ for every tree $T$, and (2)

$$
m-1+\frac{n}{2}\left(1-\frac{n}{2 \pi m}\left(\sqrt{\frac{n+4 \pi m}{n}}-1\right)\right)<\operatorname{cd}\left(K_{m}+E_{n}\right) \leq m-1+\left\lceil\frac{n}{2}\right\rceil,
$$

where $K_{m}+E_{n}$ is the join of the complete graph of order $m$ and the empty graph of order $n$. For the complete bipartite graph $K_{n, n}$ this implies $(1.286) n-1<$ $\operatorname{cd}\left(K_{n, n}\right)<(1.5) n$.
\end{abstract}

\section{Statement of the Results}

For each simple graph $G=(V, E)$, there is an embedding $f$ of $V$ into a Euclidean space $R^{n}$ such that

$$
\|f(u)-f(v)\|=1 \text { if } u v \in E \text { and }\|f(u)-f(v)\|>1 \text { if } u v \notin E \text {, }
$$

see [4]. The smallest dimension $n$ for which such an embedding exists is called the contact dimension of $G$, and is denoted by $\mathrm{cd}(G)$. Here we present some bounds on the contact dimensions of trees and of the join of a complete graph and an empty graph. For a graph $G$, let $|G|$ denote the number of vertices of $G$.

Theorem 1. For every tree $T, \mathrm{~cd}(T)<(7.3) \log |T|$.

Note that this estimate is sharp in the sense that there are trees $T$ on $n$ vertices with $\operatorname{cd}(T)>c \log n$, for some fixed $c>0$. In fact if $G$ is a graph of $n$ vertices and diameter $d$, then $\operatorname{cd}(G)>(\log n) / \log (d+1)[4$, Theorem 2]. 
Let $K_{m}+E_{n}$ be the join of the complete graph $K_{m}$ of order $m$ and the empty graph $E_{n}$ of order $n$, that is, $K_{m}+E_{n}$ is the complement of the disjoint union $E_{m} \cup K_{n}$. Define $d(m, n)=\operatorname{cd}\left(K_{m}+E_{n}\right)$. In [4] it was proved that $d(m, n) \leq$ $m-1+\lceil n / 2\rceil$ and that for any $n$, there is an $m(n)$ such that if $m>m(n)$ then $d(m, n)=m-1+\lceil n / 2\rceil$. These results are improved in the following way.

\section{Theorem 2.}

$$
m-1+\frac{n}{2}\left(1-\frac{n}{2 \pi m}\left(\sqrt{\frac{n+4 \pi m}{n}}-1\right)\right)<d(m, n) \leq m-1+\left\lceil\frac{n}{2}\right\rceil .
$$

Let us recall from [4] that, for $n \geq m, \operatorname{cd}\left(K_{m}+E_{n}\right)=\operatorname{cd}\left(K_{m, n}\right)$ holds, where $K_{m, n}$ is the complete bipartite graph. This implies:

Corollary 1. Suppose that $n \geq m$. Then

$$
m-1+\frac{n}{2}\left(1-\frac{n}{2 \pi m}\left(\sqrt{\frac{n+4 \pi m}{n}}-1\right)\right)<\operatorname{cd}\left(K_{m, n}\right) \leq m-1+\left\lceil\frac{n}{2}\right\rceil .
$$

Letting $m=n$ yields:

Corollary 2. $(1.286) n-1<d(n, n)=\operatorname{cd}\left(K_{n, n}\right)<(1.5) n$.

Since

$$
\frac{n^{2}}{4 \pi m}\left(\sqrt{\frac{n+4 \pi m}{n}}-1\right)<\frac{1}{2}
$$

is equivalent to $\left(n^{3}-n^{2}\right) / \pi<m$, we have:

Corollary 3. If $m>\left(n^{3}-n^{2}\right) / \pi$, then $d(m, n)=m-1+\lceil n / 2\rceil$.

Erdös and Füredi [2] used the probabilistic method to prove the existence of a set $X \subset R^{n}$ such that every angle spanned by three points of $X$ is acute and $|X|$ grows exponentially in $n$.

Two new proofs for this result are provided at the end of the paper, one semiconstructive and one constructive.

\section{Proof of Theorem 1}

Lemma 1. Let $O_{k}$ denote the surface area of the unit sphere in $R^{k}$, i.e., $O_{k}=$ $2 \pi^{k / 2} / \Gamma(k / 2)$. Then

$$
((k-2) /(2 \pi))^{1 / 2}<O_{k-1} / O_{k}<((k-1) /(2 \pi))^{1 / 2}
$$


Proof. Since $\log \Gamma(x)(x>0)$ is a convex function, we have

$$
\log \Gamma((k+1) / 2)+\log \Gamma((k-1) / 2)>2 \log \Gamma(k / 2)
$$

and hence $\Gamma((k+1) / 2) / \Gamma(k / 2)>\Gamma(k / 2) / \Gamma((k-1) / 2)$. Since

$$
\frac{\Gamma((k+1) / 2)}{\Gamma(k / 2)} \frac{\Gamma(k / 2)}{\Gamma((k-1) / 2)}=\frac{k-1}{2}
$$

we have

$$
\frac{\Gamma(k / 2)}{\Gamma((k-1) / 2)}<\left(\frac{k-1}{2}\right)^{1 / 2}<\frac{\Gamma((k+1) / 2}{\Gamma(k / 2)}
$$

Since $O_{k-1} / O_{k}=\Gamma(k / 2) /\left(\pi^{1 / 2} \Gamma((k-1) / 2)\right)$, we have the lemma.

Lemma 2. Let $S$ be a unit sphere in $R^{k+2}$ and $C(\alpha)$ be a spherical cap of angular radius $\alpha$. Let $|S|$ and $|C(\alpha)|$ denote the surface areas of $S$ and $C(\alpha)$. For $0<\alpha<\pi / 2$ one has

$$
\frac{|S|}{|C(\alpha)|} \geq 2\left(\frac{k}{k+1}\right)^{1 / 2} \exp \left(\frac{k(\pi / 2-\alpha)^{2}}{2}\right)
$$

Proof. Since the function $f(t)=\exp \left(t^{2} / 2\right) \cos t$ decreases on $[0, \pi / 2]$, and since

$$
|C(\alpha)|=O_{k+1} \int_{\pi / 2-\alpha}^{\pi / 2} \cos ^{k} t d t
$$

we have

$$
\begin{aligned}
|C(\alpha)| & \leq O_{k+1} \int_{\pi / 2-t}^{\pi / 2} \exp \left(\frac{-k t^{2}}{2}\right) d t \\
& <O_{k+1} \exp \left(\frac{-k(\pi / 2-\alpha)^{2}}{2}\right) \int_{0}^{\infty} \exp \left(\frac{-k t^{2}}{2}\right) d t \\
& \leq O_{k+1}\left(\frac{\pi}{2 k}\right)^{1 / 2} \exp \left(\frac{-k(\pi / 2-\alpha)^{2}}{2}\right) .
\end{aligned}
$$

Thus $|S| /|C(\alpha)|>\left(O_{k+2} / O_{k+1}\right)(2 k / \pi)^{1 / 2} \exp \left(k(\pi / 2-\alpha)^{2} / 2\right)$. Since $O_{k+2} / O_{k+1}>$ $(2 \pi /(k+1))^{1 / 2}$ by the above lemma, we have

$$
\frac{|S|}{|C(\alpha)|}>2\left(\frac{k}{k+1}\right)^{1 / 2} \exp \left(\frac{k(\pi / 2-\alpha)^{2}}{2}\right)
$$


Corollary 4. If $k+2 \geq(7.3) \log n, n \geq 3$, then $|S| /|C(\pi / 3)|>n$.

Proof. It is easy to check that if $k+2 \geq(7.3) \log n>\left(72 / \pi^{2}\right) \log n$ then $|S| /|C(\pi / 3)|>n$.

Proof of Theorem 1. Let $d$ be the smallest integer such that the ratio of the surface area of the unit sphere in $R^{d}$ and the area of the spherical cap of angular radius $60^{\circ}$ is greater than $n-2$. Then $d \leq(7.3) \log n$ by Lemma 2 . We show that $T$ is embeddable in $R^{d}$ in such a way that $\|u-v\|=1$ if $u v$ is an edge of $T$, and $>1$ otherwise. To prove this we use induction on $|T|$. It is trivial if $|T|=1$. Suppose it is true for $n-1$, and let $|T|=n$. Let $x$ be a vertex of $T$ of degree $1, T_{0}=T-\{x\}$, and let $y$ be the unique neighbor of $x$ in $T$. By induction, we can embed $T_{0}$ in $R^{d}$. Let $S$ be the unit sphere in $R^{d}$ around $y$ and $C_{1}, C_{2}, \ldots, C_{n-2}$ the intersection of the other $n-2$ unit spheres (drawn around the remaining $n-2$ vertices of $T_{0}$ ) with $S$. Clearly, $C_{i}$ is a spherical cap of angular radius $\leq 60^{\circ}$. By the choice of $d$, surf. area $(S)>\sum_{i}$ surf. area $\left(C_{i}\right)$, hence we can place $x$ on the surface of $S$ so that the distances from $x$ to all other ponts in $T_{0}$ are greater than 1 . Thus $T$ is also embeddable in $R^{d}$.

\section{Proof of Theorem 2}

A point set in Euclidean space is said to be dispersed if any two points of the set are at distance more than 1 . The following theorem was proved in [4, Theorem $6]$.

Theorem [4]. $d(m, n) \leq k+m-1$ if and only if a sphere of radius $s(m):=$ $((m+1) /(2 m))^{1 / 2}$ in $R^{k}$ contains $n$ dispersed points.

Proof of Theorem 2. Since the upper bound was proved in [4], we only show the lower bound. Suppose $k+m-1=d(m, n)$. Then, by the above theorem, a sphere $S$ of radius $s(m)$ in $R^{k}$ contains $n$ dispersed points. Let $\alpha=$ $\arcsin \left(\left(\frac{1}{2}\right)^{1 / 2} / s(m)\right)$. Since $S$ contains $n$ dispersed points, there is a spherical cap $C=C(\alpha)$ of angular radius $\alpha$ such that $C$ contains $[n|C| /|S|]$ dispersed points, where $\mid$ denotes the surface area. This can be seen in the following way. Let $x_{i}$, $i=1, \ldots, n$, be $n$ dispersed point on $S$. Consider a "random spherical cap" $C$ of angular radius $\alpha$, and define random variables $v_{i}, i=1, \ldots, n$, by $v_{i}=1$ if $x_{i} \in \mathbf{C}$, $\mathbf{v}_{i}=0$ otherwise. Then the expected value of the sum $\mathbf{v}_{1}+\cdots+\mathbf{v}_{n}$ equals $n|C| /|S|$. Hence there must be a spherical cap $C=C(\alpha)$ which contains at least $[n|C| /|S|\rceil$ dispersed points.

Next we show that if $C$ contains $k$ dispersed points then the boundary $b C$ of $C$ also contains $k$ dispersed points. Let $z \in C$ be the "center" of $C$, and let $y$ be any point of the boundary $b C$ of $C$. Then, for $m \geq 2$,

$$
\begin{aligned}
\|y-z\|^{2}=2 s(m)^{2}(1-\cos \alpha) & =((m+1) / m)\left(1-\left(1-\sin ^{2} \alpha\right)^{1 / 2}\right) \\
& =(1+1 / m)\left(1-(1 /(m+1))^{1 / 2}\right)<1
\end{aligned}
$$


Let $x_{i}, i=1, \ldots, k$, be $k$ dispersed points on $C$. Then $x_{i} \neq z$ for all $i$. For each $i$, let $y_{i}$ be the point of $b C$ such that the geodesic path on $S$ connecting $y_{i}$ and $z$ passes through $x_{i}$. (The point $y_{i}$ is the point where the great circle passing through $z$ and $x_{i}$ intersects with $b C$.)

Claim. The points $y_{i}, i=1, \ldots, k$, are dispersed.

Proof. Let $x_{j i}$ be the orthogonal projection of $x_{i}$ on the plane determined by $x_{i}$, $z$, and the center $o$ of shere $S$. Then one of the angles $\chi_{4} o x_{j i}, \Varangle z o x_{j i}$ is not less than the angle $\chi_{x_{1}} \varkappa_{j}$. Hence $\max \left\{\left\|y_{1}-x_{\mu}\right\|^{2},\left\|z-x_{j i}\right\|^{2}\right\} \geq\left\|x_{i}-x_{j i}\right\|^{2}$, and hence $\max \left\{\left\|y_{i}-x_{j}\right\|^{2},\left\|z-x_{j}\right\|^{2}\right\} \geq\left\|x_{i}-x_{j}\right\|^{2}$. However, since $\left\|z-x_{j}\right\|<1$, we have $\left\|y_{i}-x_{j}\right\|>1$ for $i \neq j$. Similarly, we can conclude $\left\|y_{i}-y_{j}\right\|>1$ for $i \neq j$. Thus $y_{i}$ $i=1, \ldots, k$, are dispersed.

Now, by Rankin's theorem [5], a sphere of radius $\leq\left(\frac{1}{2}\right)^{1 / 2}$ in $R^{k-1}$ contains at most $k$ dispersed points. Since the radius of $b C$ is $\left(\frac{1}{2}\right)^{1 / 2}, b C$ contains at most $k$ dispersed points, and so does the cap $C=C(\alpha)$ by the above argument. Hence $n|C| /|S| \leq k$.

Let us evaluate $|C| /|S|$. Since

$$
\begin{aligned}
|C| & =O_{k-1} s(m)^{k-1} \int_{0}^{\alpha}(\sin \theta)^{k-2} d \theta \\
& =O_{k-1} s(m)^{k-1}\left(\int_{0}^{\pi / 2}(\sin \theta)^{k-2} d \theta-\int_{\alpha}^{\pi / 2}(\sin \theta)^{k-2} d \theta\right)
\end{aligned}
$$

and

$$
|S|=2 O_{k-1} s(m)^{k-1} \int_{0}^{\pi / 2}(\sin \theta)^{k-2} d \theta
$$

we have

$$
\begin{aligned}
|C| /|S| & =\frac{1}{2}-\left(O_{k-1} / O_{k}\right) \int_{\alpha}^{\pi / 2}(\sin \theta)^{k-2} d \theta \\
& >\frac{1}{2}-\left(O_{k-1} / O_{k}\right)(\pi / 2-\alpha) \\
& >\frac{1}{2}-\left(O_{k-1} / O_{k}\right)(1 / m)^{1 / 2}
\end{aligned}
$$

(because $\pi / 2-\alpha<\tan (\pi / 2-\alpha)=(1 / m)^{1 / 2}$ ). Hence, by Lemma $1,|C| /|S|>$ $\frac{1}{2}-((k-1) /(2 \pi m))^{1 / 2}$. So

$$
k>n / 2-n((k-1) /(2 \pi m))^{1 / 2}>n / 2-n(k /(2 \pi m))^{1 / 2},
$$

and from this we have

$$
k>\frac{n}{2}\left(1-\frac{n}{2 \pi m}\left(\sqrt{\frac{n+4 \pi m}{n}}-1\right)\right) .
$$


Thus we have

$$
d(m, n)>\frac{n}{2}\left(1-\frac{n}{2 \pi m}\left(\sqrt{\frac{n+4 \pi m}{n}}-1\right)\right)+m-1
$$

\section{Points Without Obtuse Angle}

Erdös and Füredi [2] proved that for every $\varepsilon$ there exists a $\delta=\delta(\varepsilon)$ and points $P_{1}, \ldots, P_{m}$ on the unit sphere in $R^{d}$ so that $m>(1+\delta)^{d}$ and for all $1 \leq h<i<j \leq$ $m$ all angles of the triangle $P_{h} P_{i} P_{j}$ lie between $\pi / 3-\varepsilon$ and $\pi / 3+\varepsilon$.

Their proof is probabilistic.

Here we derive a bound using Lemma 2 .

Theorem 3. For every $0<\beta<\pi / 2$ there exist points $P_{1}, \ldots, P_{m}$ on the unit sphere $S$ in $R^{k+2}$ so that $m>(k /(k+1))^{1 / 2} \exp \left(k \beta^{2} / 2\right)$ and all distances $P_{i} P_{j}, 1 \leq i \leq j \leq m$, satisfy

$$
2(1-\sin \beta) \leq P_{i} P_{j}^{2} \leq 2(1+\sin \beta)
$$

Proof. Let $P_{1}, \ldots, P_{m}$ be a system of points on the unit sphere $S$ in $R^{k+2}$ satisfying (4.1) and such that the addition of any further point would violate (4.1). Let $D_{i}(\pi / 2-\beta)$ be the spherical double cap of angular radius $\pi / 2-\beta$ centered at $P_{i}$ (a double cap is the union of two diametrically opposite caps). Then the union of $D_{i}(\pi / 2-\beta)$ for $i=1, \ldots, m$ has to cover all points on the sphere. (In fact, if $Q \notin D_{i}(\pi / 2-\beta)$ then by elementary computation

$$
2(1-\sin \beta)<P_{i} Q^{2}<2(1+\sin \beta)
$$

holds.) However, $\left|D_{i}(\pi / 2-\beta)\right|=2|C(\pi / 2-\beta)|$ and Lemma 2 yields

$$
m \geq|S| /(2|C(\pi / 2-\beta)|) \geq(k /(k+1))^{1 / 2} \exp \left(k \beta^{2} / 2\right),
$$

as desired.

Remark. Note that the proof of Theorem 3 shows that every maximal (nonextendable) set satisfying (4.1) is exponentially large. Thus one can construct such a set by adding the points one by one.

Corollary 5. There exist points $P_{1}, \ldots, P_{m} \in S \subset R^{k+2}$ so that all triangles $P_{h} P_{i} P_{j}$, $1 \leq h<i<j \leq m$, are acute and

$$
m \geq(1.0594)^{k}(k /(k+1))^{1 / 2}
$$

holds. 
Proof. It is sufficient to choose $\beta$ so that $\sin \beta=\frac{1}{3}$ and apply Theorem 3 .

Corollary 6. There are points $P_{1}, \ldots, P_{m} \in S \subset R^{k+2}$ so that all triangles $P_{h} P_{i} P_{j}$, $1 \leq h<i<j \leq m$, have all angles between $59^{\circ}$ and $61^{\circ}$ and $m \geq$ $(1.00011)^{k}(k /(k+1))^{1 / 2}$ holds.

Proof. This time one chooses $\beta$ so that $\sin 30.5^{\circ}=\frac{1}{2}((1+\sin \beta) /(1-\sin \beta))^{1 / 2}$ (for this value of $\beta$ one checks that $\sin 29.5^{\circ}<\frac{1}{2}((1-\sin \beta) /(1+\sin \beta))^{1 / 2}$ holds) and applies Theorem 3 .

In [2] somewhat better bounds are obtained both in Corollaries 5 and 6 . The reason is that Erdös and Füredi choose the points from the $2^{n}$ vertices of the cube which span no obtuse angles. Thus in the case of Corollary 5 one has to get rid of right angles only. Let us recall the standard correspondence between the vertices of the $n$-cube and the subsets of an $n$-element set $X$. By Pythagoras' theorem (see [2]) three vertices corresponding to subsets $A, B, C \subset X$ span a right angle at $C$ if and only if $A \cap B \subset C \subset A \cup B$ holds.

One can use a recent result of Friedman [3] to obtain an explicit construction for such a family of exponential size (and, consequently, of exponentially many vertices of the $n$-cube with all angles acute). In fact, a special case of Friedman's [3] Theorem 5.7 gives an explicit construction for more than $59^{d}$ sequences of 59 symbols and of length $10^{32} d$ so that for any three sequences there is at least one place where all three are different. (This result of Friedman was used by Alon [1] to obtain explicit construction for other related families of exponential size.)

Now let $b$ be the smallest integer so that there exists a family $\left\{F_{1}, \ldots, F_{59}\right\}$ of subsets of $\{1,2, \ldots, b\}$ without three sets $F_{h}, F_{l}, F_{j}$ satisfying $F_{h} \cap$ $F_{i} \subset F_{j} \subset F_{h} \cup F_{i}$ (to be more explicit one can also take $b=59$ and $F_{i}=\{i\}$ ). Then in each sequence replace each appearance of the $i$ th symbol by a $(0,1)$-sequence of length $b$ corresponding to $F_{i}$. With $n=10^{32} b d$ this gives $59^{d}>2^{5 n / c}$ (where $c=10^{32} b$ ), i.e., exponentially many vertices of the $n$-cube without right angles.

Finally, let us call the reader's attention to a forthcoming interesting paper of Reiterman et al. [6] on sphericity and another related dimension of graphs.

\section{Acknowledgment}

The authors are indebted to the referees for valuable suggestions.

\section{References}

1. N. Alon, Explicit construction of exponeitial-sized families of $k$-independent sets, Discrete Math. 58 (1986), 191-193.

2. P. Erdös and Z. Füredi, The greatest angle among $n$ points in the $d$-dimensional euclidean space, Ann. Discrete Math. 17 (1983), 275-283. 
3. J. Friedman, Constructing $O(n \log n)$ size monotone formula for the $k$ th elementary symmetric polynomial of $n$ Boolean variables, Proceedings of the 25th IEEE Symposium on Foundations of Computer Science, 506-515, 1984.

4. H. Maehara, Contact patterns of equal nonoverlapping spheres, Graphs Combin. 1 (1985).271-282.

5. R. A. Rankin, The closest packing of spherical caps in n-dimensions, Proc. Glasgow Math. Assoc. 2 (1955) 139-144.

6. J. Reiterman, V. Rödl, and E. Siňajová, Geometrical embeddings of graphs, Discrete Math., to appear.

Received March 17, 1986, and in revised form August 1, 1986. 